\title{
Uncertainty estimation of low voltage LI measurements with recurrent surge generator and oscilloscope
}

Zdenko Godec $^{1}$, Vjenceslav Kuprešanin ${ }^{1}$ and Filip Razum ${ }^{1}$

${ }^{1}$ Končar - Electrical Engineering Institute, Fallerovo Šetalište 22, HR-10000 Zagreb, Croatia

E-mail: vkupresa@koncar-institut.hr

Abstract — Low voltage LI measurement with recurrent surge generator and oscilloscope is a method used for investigating LI distribution in transformer windings or in model prototypes for various conditions. In the paper, the procedure for measurement uncertainty estimation of characteristic voltage and time quantities is given. The procedure is similar to the procedure given in the IEC 60076-2 standard for high voltage testing, but it is fully adapted to low voltage measurement with recurrent generator and oscilloscope. For the purpose of reducing the measurement uncertainties and the measurement time, a new procedure is proposed.

Index Terms-Low voltage lightning impulse measurement, measurement uncertainty, complete measurement result, stability, repeatability, nonlinearity

\section{INTRODUCTION}

Low voltage lightning impulse (LI) measurement with recurrent surge generator and oscilloscope is a non-destructive, widely accepted method used in the transformer industry. The method is primarily used for investigating LI voltage distribution in transformer windings or in model prototypes for various impulse voltage conditions, giving useful information for proper insulation design. In addition, it can be used for estimating the impulse circuit parameters for LI testing or as a control for transient calculation tools. In this method, the recurrent surge generator acts as a low voltage equivalent of the high voltage impulse generator, and the oscilloscope is used for measurement and analysis. As an input voltage, most often standard lightning-impulse voltage, described as $1,2 \mu \mathrm{s} / 50 \mu \mathrm{s}$ impulse, or standard chopped lightning impulse voltage (chopping in range $2 \mu \mathrm{s}$ to $5 \mu \mathrm{s}$ ) is used. The main measurement results are the input voltage with its parameters $\left(U_{\mathrm{p}}\right.$ - peak voltage $(\mathrm{V}), T_{1}$ - front time $(\mu \mathrm{s}), T_{2}$ - time to half-value $(\mu \mathrm{s}), T_{\mathrm{c}}$ - time to chopping $(\mu \mathrm{s}), \beta^{\prime}-$ relative overshoot magnitude (\%)) and the response voltages measured in different points, regularly expressed in percentage of the input voltage.

In the paper, the uncertainty estimates for low voltage parameters measured by the usual procedure are given. Usual procedure assumes simultaneous recording of the input (reference) voltage and one or more response voltages. Based on the uncertainty component analysis of the usually used procedure, an improved measurement procedure is proposed. The new procedure reduces both the measurement uncertainties and the time required to complete measurements.

A new software for data acquisition and lightning impulse analysis is developed in National Instruments ${ }^{T M}$ Labview programming language. Impulse parameters are calculated according to the procedure given in [1], and the algorithm is verified based on [2]. All measurement results, presented in the tables, were obtained with this application. Although the procedure for determination of impulse voltage parameters is primarily introduced for high voltage impulse testing, it is reasonable to apply the same algorithm for low voltage measurements, making it a better simulation and control tool.

II. BLOCK DIAGRAM AND MATHEMATICAL MODEL OF MEASUREMENT

In Fig. 1., a simplified block diagram of measurement is shown.

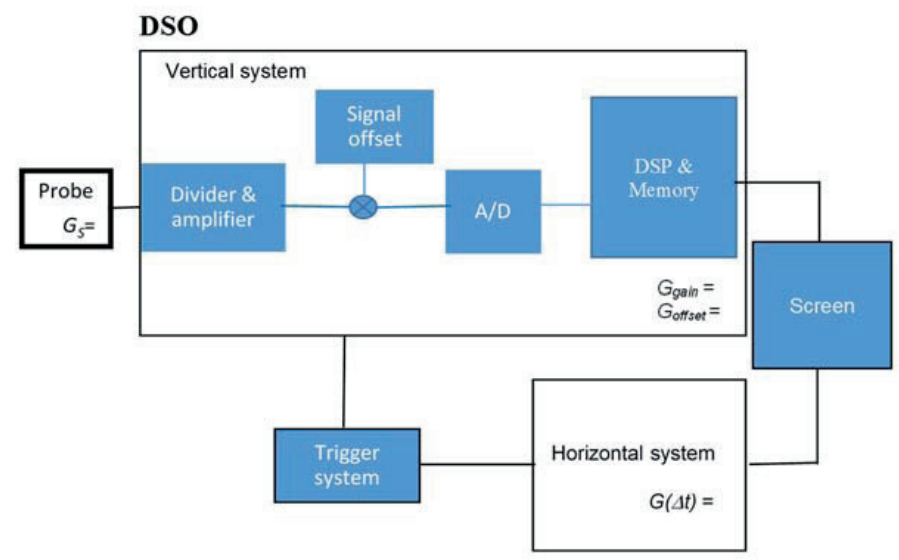

Figure 1: Simplified block diagram of the measurement system (digital oscilloscope + probe)

A voltage to be measured is applied to the input of the probe. The probe output is connected to the input of the digital oscilloscope. On the oscilloscope screen, voltage $V_{\mathrm{M}}$ and time interval $\Delta t$ are read (graphically or digitally): 


$$
\begin{aligned}
& V_{M}=k_{S} \cdot k_{V O S} \cdot V=n_{V} \cdot d_{V} \\
& \Delta t=n_{t} \cdot d_{t}
\end{aligned}
$$

Symbol $k_{\mathrm{S}}$ represents measuring probe scale factor (mostly voltage damping), $k_{\mathrm{V} O \mathrm{~s}}$ is a factor that includes digital signal processing (DSP) of an oscilloscope vertical system, $n_{\mathrm{V}}$ is a number of divisions in the vertical direction and $d_{\mathrm{V}}$ is (adjusted) sensitivity of the vertical scale, expressed in volts per division (V/div). The sensitivity in vertical direction also includes the nominal factor of the measuring probe, so that the oscilloscope directly shows (graphically and digitally) the measured voltage $V_{\mathrm{M}}$.

Further, $n_{\mathrm{t}}$ is a number of divisions (div) in the horizontal direction, and $d_{\mathrm{t}}$ is (adjusted) sensitivity of the horizontal scale, expressed in seconds per division (s/div).

\section{STANDARD MEASUREMENT UNCERTAINTY OF VOLTAGE}

Based on the first part of the mathematical model (1), the expression for standard measurement uncertainty of peak-topeak voltage is derived:

$$
u\left(V_{M}\right)_{\%}=\sqrt{\left[u\left(k_{S}\right)_{\%}\right]^{2}+\left[u\left(k_{V O S}\right)_{\%}\right]^{2}+u_{\% \text { res }}^{2}}
$$

where $u_{\%}$ res is the uncertainty component due to the oscilloscope finite resolution.

Standard measurement uncertainties are estimated based on specified error limits (accuracy) [3].

For measuring probes, percentage error limits with respect to measured voltage are given. E.g., for probe type P6139B [4] error limits are:

$$
G_{\% S}= \pm 0,5 \%
$$

Percentage standard uncertainty of voltage at the output of measuring probe is then [3]:

$$
u\left(k_{S}\right)_{\%}=\frac{G_{\% S}}{\sqrt{3}}
$$

The way of specifying the error limits for the oscilloscopes is not standardized, so the form of specification mostly depends on the manufacturer.

For the vertical system of the oscilloscope TEKTRONIX TDS7104, the next specification is given [5]:

a) Error limit of the attenuation (scale factor) with respect to the measured value:

$$
G_{\% \text { gain }}= \pm 1 \%
$$

consequently, the absolute error limit is:

$$
\dot{G}_{\text {gain }}= \pm \frac{1}{100} \cdot n_{V} \cdot d_{V}
$$

$n_{\mathrm{v}}$ is measured number of divisions, and $d_{\mathrm{v}}$ is sensitivity in the vertical direction ( $/$ div).

b) Absolute error limit of the offset:

$$
\dot{G}_{\text {Offset }}= \pm\left(0,25 \% \times \mid\left(\text { offset }- \text { Position } \times \frac{\mathrm{v}}{\operatorname{div}}\right) \mid+150 \mathrm{mV}+0,1 \operatorname{div} \times \frac{\mathrm{v}}{\operatorname{div}} \text { setting }\right)
$$

where Offset is adjusted offset voltage $V_{\text {Offset }}$, Position is a channel reference position with respect to the vertical scale central line (div), $n_{\mathrm{Vc}}$, multiplied with adjusted sensitivity $d_{\mathrm{V}}(\mathrm{V} / \mathrm{div})$.

Absolute error limit of the offset can be more clearly stated as follows:

$$
\dot{G}_{\text {Offset }}= \pm\left[0,25 \% \times\left|\left(V_{\text {offset }}-n_{V c} \cdot d_{V}\right)\right|+150 m \mathrm{~V}+0,1 \mathrm{div} \times d_{V}\right]
$$

Error limit for each of the voltage samples (measuring points) is defined as:

$$
\begin{gathered}
\dot{G}(V)= \pm\left[1 \% \times \mid \text { reading }-\left(\text { Offset }- \text { Position } \times \frac{\mathrm{v}}{\mathrm{div}}\right) \mid+ \text { Offset Accuracy }+\right. \\
\left.+0,13 \operatorname{div} \times \frac{\mathrm{v}}{\operatorname{div}} \text { setting }+0,6 \mathrm{mV}\right]
\end{gathered}
$$

For this type of the oscilloscope, absolute error limit of peak-to-peak voltage (Vpp) is also defined:

$$
\dot{G}\left(V_{p p}\right)= \pm\left[1 \% \times \mid \text { reading } \mid+0,26 \frac{\mathrm{V}}{\text { div }} \text { setting }+1,2 \mathrm{mV}\right]
$$

or more clearly:

$$
\dot{G}\left(V_{p p}\right)= \pm\left[\frac{1 \%}{100 \%} \times\left|n_{V} \cdot d_{V}\right|+0,26 \operatorname{div} \cdot d_{V}+1,2 \mathrm{mV}\right]
$$

Absolute standard uncertainty of measured voltage is then:

$$
\dot{u}\left(k_{V O S}\right)=\frac{\dot{G}\left(V_{p p}\right)}{\sqrt{3}}
$$

with percentage standard uncertainty being equal to:

$$
u\left(k_{V O S}\right)_{\%}=\frac{\dot{u}\left(k_{V O S}\right)}{n_{V} \cdot d_{V}} 100 \%
$$


Finally, percentage standard uncertainty of voltage measurement (probe + oscilloscope) is estimated as:

$$
u\left(V_{M}\right)_{\%}=\sqrt{\left[\frac{G \% S}{\sqrt{3}}\right]^{2}+\left[\frac{100 \%}{n_{V} \cdot d_{V}} \cdot \frac{\dot{G}\left(V_{p p}\right)}{\sqrt{3}}\right]^{2}+u_{\% \text { res }}^{2}}
$$

and the absolute standard uncertainty is:

$$
\dot{u}\left(V_{M}\right)=\frac{u\left(V_{M}\right) \%}{100 \%} \cdot V_{M}
$$

Component of the standard uncertainty (absolute) due to the oscilloscope finite resolution is equal:

$$
\dot{u}_{\text {res }}=\frac{\dot{R}}{2 \cdot \sqrt{3}}=\frac{d_{v} \cdot 10 \mathrm{div}}{2 \cdot \sqrt{3} \cdot 2^{x}}
$$

where $\dot{R}$ is the resolution, and $x$ is the effective number of bits (ENOB).

Percentage component of the standard uncertainty due to the finite resolution is calculated as:

$$
u_{\% \text { res }}=\frac{10 \cdot 100 \%}{2 \cdot \sqrt{3} \cdot 2^{x} \cdot n_{v}}=\frac{10 \cdot d_{v} \cdot 100 \%}{2 \cdot \sqrt{3} \cdot 2^{x} \cdot V_{M}}
$$

\section{STANDARD MEASUREMENT UNCERTAINTY OF VOLTAGE RATIO}

As a rule in low voltage LI measurement reports, peak values of the measured response voltages are expressed in percentage of the applied lightning impulse $V_{0}$ (its peak value):

$$
v_{\% i}=\frac{v_{i}}{V_{0}} 100 \%
$$

The standard measurement uncertainty of voltage ratio (\%) is:

$$
u\left(v_{\% i}\right)_{\%}=\sqrt{\left[u\left(V_{i}\right)_{\%}\right]^{2}+\left[u\left(V_{0}\right)_{\%}\right]^{2}}
$$

Since the voltage ratio is a quantity given in percentage, it is more convenient to calculate the absolute standard uncertainty:

$$
\dot{u}\left(v_{\% i}\right)=\frac{u\left(v_{\% i}\right)_{\%}}{100 \%} \cdot v_{\% i}
$$

V. STANDARD MEASUREMENT UNCERTAINTY OF TIME INTERVAL

Error limits of measured time interval are also specified in different ways by different manufacturers.

Expression (22) explains the relationship among different characteristic time quantities of the oscilloscope:

$$
f_{U}=\frac{R L}{\Delta t_{D}}
$$

where symbols are:

$f_{U}-$ sample rate in $\mathrm{S} / \mathrm{s}$ (Samples per second),

$R L$ - record length expressed as the number of samples stored in memory,

$\Delta t_{D}$ - time domain or range in seconds (equal to number of divisions in horizontal direction $\left(n_{t D}\right)$ multiplied with scale sensitivity $d_{t} ; n_{t D}$ is mostly equal 10 ,

$d_{t}-$ sensitivity of the horizontal (time) scale in s/div.

Time resolution is better with higher sampling frequency $\left(t_{\mathrm{sr}}=1 /(\right.$ Sample rate $\left.)\right)$. Therefore, the time sensitivity and the length of the record should be adjusted to achieve the highest possible sampling frequency with noise (interferences) still being acceptable.

For the oscilloscope Tektronix TDS7104 the manufacturer for the time interval measurement $(\Delta t=D T)$ specifies the following expression for the error limit $(D T A=$ Delta Time Accuracy):

$$
D T A=\dot{G}(\Delta t)= \pm\left(15 \text { ppm } x \mid \text { reading } \mid+\frac{0,3}{\text { real time sample rate }}\right)
$$

The absolute standard uncertainty (in seconds) is estimated as:

$$
\dot{u}(\Delta t)=\frac{\dot{G}(\Delta t)}{\sqrt{3}}
$$

and the percentage standard uncertainty of time interval $\Delta t$ is:

$$
u(\Delta t)_{\%}=\frac{\dot{u}(\Delta t)}{\Delta t} \cdot 100 \%
$$




\section{INFLUENCING FACTORS}

The IEC standard [1] specifies an expression for estimating the extended relative measurement uncertainty of a measured voltage $V_{\mathrm{M}}$ which contains a number of components of measurement uncertainty caused by different influencing factors:

$$
U_{M}=k \cdot u_{M}=2 \sqrt{u_{c a l}^{2}+\sum_{i=0}^{N} u_{B i}^{2}}
$$

$u_{\text {cal }}$ is the standard uncertainty of scale factor of the approved measuring system determined by the calibration procedure, $u_{\mathrm{Bi}}$ is the contribution to the combined standard uncertainty of the scale factor of the approved measuring system, caused by the i-th influence quantity and evaluated as a Type B contribution. These contributions are related to normal use of the approved measuring system, and arise from non-linearity, short-term and long-term instabilities, etc. and are determined by additional measurements or are estimated from other data sources. Other significant influences shall be taken into account, e.g. resolution of instrument display - if it is significant.

Effect of nonlinearity is expressed by relative standard uncertainty $u_{\mathrm{B} 0}$, effect of extension of the validity of the scale factor is expressed by $u_{\mathrm{B} 1}$ (not relevant in our measurements), effect of dynamic behavior (amplitude/frequency response) is expressed by $u_{\mathrm{B} 2}$, effect of short-term stability by $u_{\mathrm{B} 3}$, effect of long-term stability by $u_{\mathrm{B} 4}$, effect of ambient temperature by $u_{\mathrm{B} 5}$, proximity effect by $u_{\mathrm{B} 6}$, and software effect by $u_{\mathrm{B} 7}$.

All of the above-listed uncertainty components are covered by the specified error limits of the measuring probe and the oscilloscope, provided that the measurements are made in the temperature range from $10{ }^{\circ} \mathrm{C}$ to $45{ }^{\circ} \mathrm{C}[5]$ and that a negligible proximity effect is provided during the measurement. Only software effect uncertainty $u_{\mathrm{B} 7}$, upper limits of which are given in table 3 in [2] and abbreviated here in table I., is not covered by the error limits of the measuring channels.

TABLE I. STANDARD UNCERTAINTY CONTRIBUTIONS OF SOFTWARE $\left(u_{\% \text { soft }}\right)$ TO THE
OVERALL UNCERTAINTY ACCORDING TO THE SIMPLIFIED PROCEDURE [2]
\begin{tabular}{ccccc}
\hline $\begin{array}{c}\text { Inpulse } \\
\text { group/evaluation } \\
\text { algorithm }\end{array}$ & $V / \%$ & $B / \%$ & $T_{1} / \%$ & $T_{2} / \%$ \\
\hline LI & 0,058 & 0,58 & 1,2 & 0,58 \\
\hline LIC & 0,58 & 0,58 & 1,2 & 1,2 \\
\hline SI & 0,29 & - & 2,9 & 1,2 \\
\hline
\end{tabular}

It means that for the total (overall) uncertainty, to previously calculated standard uncertainties (chapters II to V) corresponding uncertainties from table I. should be added.

\section{COMPLETE MEASUREMENT RESULT OF VOLTAGE RATIO AND TIME INTERVAL WITH STANDARD PROCEDURE}

\section{A. Complete measurement result of voltage ratio}

Nowadays, it is common for the complete measurement result to be expressed by the extended measurement uncertainty with $k=2$. Extended measurement uncertainty increases the confidence that the true value of a measured quantity is within the range of values determined by the measured value and expanded uncertainty (approximately 95\% for $k=2$ ) [3].

The complete measurement result for voltage ratio is then:

$$
v_{\% i}=v_{\% i} \pm 2 \cdot \dot{u}_{\mathrm{T}}\left(v_{\% i}\right)
$$

In the final result, the expanded measurement uncertainty is rounded to two significant digits, and the standard measurement uncertainties (as intermediate results) are rounded to four significant digits.

The total (overall) uncertainty of the voltage ratio (the response voltage expressed as a percentage of the applied voltage) is estimated by the expression:

$$
\dot{u}_{\mathrm{T}}\left(v_{\% i}\right)=\sqrt{\dot{u}\left(v_{\% i}\right)^{2}+2 \cdot u_{\% \text { soft }}^{2}}
$$

\section{EXAMPLE 1}

With the HAEFELY Type 481 generator [6], a peak-to-peak voltage of 300,3 V was applied. The measurement was performed by TEKTRONIX P6139B passive probe [4] and the TEKTRONIX TDS7104 oscilloscope [5]. Offset was 0 V, and the sensitivity of the vertical system was adjusted to $d_{V}=50 \mathrm{~V} /$ div.

The response peak to peak voltage between two points was $46,66 \mathrm{~V}$, measured by another measuring channel (oscilloscope + measuring probe) with vertical sensitivity $d_{V}=10 \mathrm{~V} /$ div.

The measurement uncertainty of the response voltage ratio should be estimated.

According to (12), the error limit of the oscilloscope when measuring the applied voltage (300,3 V) is: 


$$
\begin{aligned}
\dot{G}\left(V_{p p}\right)= \pm & {\left[\frac{1 \%}{100 \%} \times\left|n_{V} \cdot d_{V}\right|+0,26 \operatorname{div} \cdot d_{V}+1,2 \mathrm{mV}\right]=} \\
= & \pm\left[\frac{1 \%}{100 \%} \cdot 300,3+0,26 \cdot 50+1,2 \mathrm{mV}\right]=16,00 \mathrm{~V}
\end{aligned}
$$

The absolute standard measurement uncertainty of voltage measured by the oscilloscope is estimated according to (13):

$$
\dot{u}\left(k_{V O S}\right)=\frac{\dot{G}\left(V_{p p}\right)}{\sqrt{3}}=\frac{16,00}{\sqrt{3}}=9,238 \mathrm{~V}
$$

and the percentage standard uncertainty is (14):

$$
u\left(k_{V O S}\right)_{\%}=\frac{\dot{u}\left(k_{V O S}\right)}{n_{V} \cdot d_{V}} 100 \%=\frac{9,238}{300,3} 100 \%=3,078 \%
$$

Component of the uncertainty due to the oscilloscope finite resolution (expressed in percentage) is estimated by the expression (18):

$$
u_{\% \text { res }}=\frac{10 \cdot d_{v^{\prime}} \cdot 100 \%}{2 \cdot \sqrt{3} \cdot 2^{x} \cdot V_{M}}=\frac{10 \cdot 50 \cdot 100 \%}{2 \cdot \sqrt{3} \cdot 2^{8,7} \cdot 300,3}=0,1156 \%
$$

According to the manufacturer's specification, the effective number of bits (ENOB) of the oscilloscope type TDS7104 is $\mathrm{x}=8,7$ when using Hi-Res acquisition.

The percentage standard uncertainty of measured applied voltage (300,3 V; probe + oscilloscope) is estimated by the expression (15):

$$
\begin{aligned}
u\left(V_{M}\right)_{\%} & =\sqrt{\left[\frac{G_{\% S}}{\sqrt{3}}\right]^{2}+\left[\frac{100 \%}{n_{V} \cdot d_{V}} \cdot \frac{\dot{G}\left(V_{p p}\right)}{\sqrt{3}}\right]^{2}+u_{\% \text { res }}^{2}}= \\
& =\sqrt{\left[\frac{0,5}{\sqrt{3}}\right]^{2}+\left[\frac{100 \%}{300,3} \cdot \frac{16,00}{\sqrt{3}}\right]^{2}+0,1156^{2}}=3,092 \%
\end{aligned}
$$

The same procedure applies when estimating the measurement uncertainty of the response voltage (46,66 V). According to (12), the error limit of the oscilloscope when measuring the response voltage is:

$$
\begin{aligned}
\dot{G}\left(V_{p p}\right)= \pm\left[\frac{1 \%}{100 \%} \times\left|n_{V} \cdot d_{V}\right|+0,26 \mathrm{div} \cdot d_{V}+1,2 \mathrm{mV}\right] & = \\
= \pm\left[\frac{1 \%}{100 \%} \cdot 46,66+0,26 \cdot 10+1,2 \mathrm{mV}\right] & =3,068 \mathrm{~V}
\end{aligned}
$$

The absolute standard uncertainty of the oscilloscope due to its error limit is (13):

$$
\dot{u}\left(k_{V O S}\right)=\frac{\dot{G}\left(V_{p p}\right)}{\sqrt{3}}=\frac{3,068}{\sqrt{3}}=1,771 \mathrm{~V}
$$

and the percentage uncertainty is (14):

$$
u\left(k_{V O S}\right)_{\%}=\frac{\dot{u}\left(k_{V O S}\right)}{n_{V} \cdot d_{V}} 100 \%=\frac{1,771}{46,66} 100 \%=3,796 \%
$$

Percentage component of the uncertainty due to the oscilloscope finite resolution is equal to (18):

$$
u_{\% \text { res }}=\frac{10 \cdot d_{v} \cdot 100 \%}{2 \cdot \sqrt{3} \cdot 2^{x} \cdot V_{M}}=\frac{10 \cdot 10 \cdot 100 \%}{2 \cdot \sqrt{3} \cdot 2^{8,7} \cdot 46,66}=0,1488 \%
$$

Percentage standard uncertainty of response voltage measurement (46,66 V; probe + oscilloscope) is (15):

$$
\begin{gathered}
u\left(V_{M}\right)_{\%}=\sqrt{\left[\frac{G \% S}{\sqrt{3}}\right]^{2}+\left[\frac{100 \%}{n_{V} \cdot d_{V}} \cdot \frac{\dot{G}\left(V_{p p}\right)}{\sqrt{3}}\right]^{2}+u_{\% \text { res }}^{2}}= \\
=\sqrt{\left[\frac{0,5}{\sqrt{3}}\right]^{2}+\left[\frac{100 \%}{46,66} \cdot \frac{3,068}{\sqrt{3}}\right]^{2}+0,1488^{2}}=3,810 \%
\end{gathered}
$$

Percentage standard uncertainty of voltage ratio is estimated by the expression (20):

$$
u\left(v_{\% i}\right)_{\%}=\sqrt{\left[u\left(V_{i}\right)_{\%}\right]^{2}+\left[u\left(V_{0}\right)_{\%}\right]^{2}}=\sqrt{3,092^{2}+3,810^{2}}=4,907 \%
$$

with the absolute standard uncertainty of voltage ratio being (21):

$$
\dot{u}\left(v_{\% i}\right)=\frac{u\left(v_{\% i}\right)_{\%}}{100 \%} \cdot v_{\% i}=\frac{4,907 \%}{100 \%} \cdot \frac{46,66}{300,3} 100 \%=0,7624 \%
$$

Total (overall) uncertainty of voltage ratio (i.e. the response voltage expressed in a percentage of applied voltage) is estimated according to (28):

$$
\dot{u}_{\mathrm{T}}\left(v_{\% i}\right)=\sqrt{\dot{u}\left(v_{\% i}\right)^{2}+2 \cdot u_{\% \text { soft }}^{2}}=\sqrt{0,7624^{2}+2 \cdot 0,058^{2}}=0,7668 \%
$$

Complete measurement result of voltage ratio is according to (27):

$$
v_{\% i}=v_{\% i} \pm 2 \cdot \dot{u}_{\mathrm{T}}\left(v_{\% i}\right)=\frac{46,66}{300,3} 100 \% \pm 2 \cdot 0,7668 \%=\{15,5 \pm 1,5\} \%
$$




\section{B. Complete measurement result of time interval}

The overall percentage uncertainty of time interval is estimated by:

$$
u_{\mathrm{T}}(\Delta t)_{\%}=\sqrt{u(\Delta t)_{\%}^{2}+2 \cdot u_{\% \text { soft }}^{2}}
$$

and the complete measurement results of time interval is then:

$$
\Delta t=\Delta t\left[1 \pm 2 \cdot u_{\mathrm{T}}(\Delta t)_{\%}\right] \mathrm{s}
$$

\section{EXAMPLE 2}

For the standard lightning impulse voltage from example 1, time to half-value $\left(T_{2}\right)$ of $56,7 \mu$ s is measured. The horizontal scale sensitivity and record length were $10,0 \mu \mathrm{s} /$ div and $\mathrm{RL}=2500$ respectively. Uncertainty of time interval measurement $\left(\Delta t=T_{2}\right)$ should be estimated.

Absolute error limit (in seconds) of time interval measurement by the oscilloscope is according to (23):

$$
\begin{aligned}
\dot{G}(\Delta t)= & \pm\left(15 \text { ppm } x \mid \text { reading } \mid+\frac{0,3}{\text { real time sample rate }}\right)= \\
& = \pm\left(15 \cdot 10^{-6} x\left|56,7 \cdot 10^{-6}\right|+\frac{0,3 \cdot 100 \cdot 10^{-6}}{2500}\right)=1,285 \cdot 10^{-8} \mathrm{~s}
\end{aligned}
$$

The absolute standard uncertainty (in seconds) is (24):

$$
\dot{u}(\Delta t)=\frac{\dot{G}(\Delta t)}{\sqrt{3}}=\frac{0,01285 \mu \mathrm{s}}{\sqrt{3}}=0,007419 \mu \mathrm{s}
$$

and the percentage standard uncertainty of time interval measurement by the oscilloscope is (25):

$$
u(\Delta t)_{\%}=\frac{\dot{u}(\Delta t)}{\Delta t} 100 \%=\frac{0,007419}{56,7} 100 \%=0,01308 \%
$$

The total (overall) uncertainty of time interval measurement is estimated by the expression (29):

$$
u_{\mathrm{T}}(\Delta t)_{\%}=\sqrt{u_{\mathrm{T}}(\Delta t)_{\%}^{2}+2 \cdot u_{\% \text { soft }}^{2}}=\sqrt{0,01308^{2}+2 \cdot 1,2^{2}}=1,697 \%
$$

The complete measurement result of time to half-value $\left(T_{2}\right)$ is according to (30):

$$
T_{2}=56,7[1 \pm 3,4 \%]
$$

\section{NEW PROCEDURE FOR LOW VOLTAGE LI MEASUREMENT}

The uncertainties analyzed in previous sections are in accordance with the old (standard) procedure of low voltage LI measurements. In this procedure, the applied voltage, $V_{\mathrm{o}}$, is measured by one measuring channel and the response voltages, $V_{i}$, are simultaneously (synchronously) measured on the remaining channels.

Given that the estimated expanded measurement uncertainty of the voltage ratio is quite large, we changed the measurement procedure. In the new procedure, the applied voltage, $V_{0}$, is adjusted and then measured by all available measuring channels. In the second step (and subsequent steps) only the response voltages, $V_{i}$, are measured by available measuring channels. It means that the applied voltage and response voltage are measured by the same measuring channel, but not simultaneously. As the applied and response voltages are measured by the same channel, they are in a functional relationship, or, more precisely, voltages are correlated due to the instability of the recurrent surge generator, the channels instability, resolution and nonlinearity of the measuring channels, and random deviations (interferences).

The measuring channels need not be calibrated, since both voltages (applied and response) are measured on the same measuring scale.

In order to verify the assumptions, it was first necessary to experimentally verify that the recurrent surge generator and the measuring channels are sufficiently stable within a given period of time, i.e. it was necessary to estimate (measure) the repeatability of the measurements over a period of 48 hours. The results are listed in Table II.

Table II. shows that the measuring channels measure the same voltage differently (error limits), but the standard deviations for each measuring channel are much smaller (due to stability of the generator and measuring channels).

Nonlinearity is the next influencing factor that needs to be checked and for which the component of measurement uncertainty has to be estimated. The usual range of measured response voltages is $15 \%$ to $100 \%$, and the applied voltage is typically $300 \mathrm{~V}$. In order to check the nonlinearity of the generator and the measuring channels, we adjusted the surge generator voltage to $50 \mathrm{~V}, 100 \mathrm{~V}, 150 \mathrm{~V}, 200 \mathrm{~V}, 250 \mathrm{~V}$ and $300 \mathrm{~V}$ and measured it simultaneously with the HIGHVOLT HiRES digital transient recorder (as reference) and the measuring channels of our measuring system (oscilloscope). All measurements were repeated 10 times and the measurement uncertainties were estimated according to [1]. The results are shown in Table III.

$\bar{F}_{1, i}$ is the mean of 10 repeated measurements $\left(j ; j_{\max }=n=10\right)$ of the scale factor $\left(V_{\mathrm{HiRES}} / V_{1}\right)$ of channel 1 at the , $i$ " voltage level $\left(i ; i_{\max }=m=6\right)$ and $u_{1, i}$ is the relative standard deviation of the mean value. 


$$
\begin{aligned}
& \bar{F}_{1, i}=\frac{1}{n} \sum_{j=1}^{n}\left(\frac{V_{\mathrm{HiRES}}}{V_{1 ; j}}\right) \\
& u_{1, i}=\frac{1}{\bar{F}_{1, i}} \sqrt{\frac{1}{n \cdot(n-1)} \sum_{j=1}^{n}\left(F_{1, i ; j}-\bar{F}_{1, i}\right)^{2}}
\end{aligned}
$$

$F$ is the mean of the scale factor across all voltage levels $\left(i, i_{\max }=m=6\right)$ :

$$
F=\frac{1}{m} \sum_{i=1}^{m} \bar{F}_{1, i}
$$

$u_{1, B O}$ is the relative standard uncertainty due to nonlinearity for channel 1:

$$
u_{1, B O}=\frac{1}{\sqrt{3}} \max _{i=1}^{m}\left|\frac{\bar{F}_{1, i}}{F}-1\right|
$$

The same applies to the remaining measuring channels.

Relative standard uncertainty due to nonlinearity for all four channels, $u_{\mathrm{F}}$, is calculated as pooled standard deviation:

$$
u_{F}=\sqrt{\frac{\sum_{k=1}^{4}\left(m_{k}-1\right) u_{k, B O}^{2}}{\sum_{k=1}^{4}\left(m_{k}-1\right)}}=\frac{1}{2} \sqrt{\sum_{k=1}^{4} u_{k, B O}^{2}}
$$

\begin{tabular}{|c|c|c|c|c|}
\hline Parameters & $\begin{array}{c}\text { Measuring } \\
\text { channels }\end{array}$ & Mean & Standard deviation & No. of measurements \\
\hline \multirow{4}{*}{$V_{0}$} & 1 & $302,52 \mathrm{~V}$ & $0,06725 \%$ & 51 \\
\hline & 2 & $300,76 \mathrm{~V}$ & $0,1067 \%$ & 51 \\
\hline & 3 & $301,73 \mathrm{~V}$ & $0,05860 \%$ & 51 \\
\hline & 4 & $301,27 \mathrm{~V}$ & $0,1103 \%$ & 51 \\
\hline \multirow{4}{*}{$\beta$} & 1 & $-0,24012 \%$ & $0,04652 \%$ & 51 \\
\hline & 2 & $-0,24778 \%$ & $0,04967 \%$ & 51 \\
\hline & 3 & $-0,21083 \%$ & $0,04427 \%$ & 51 \\
\hline & 4 & $-0,18255 \%$ & $0,04516 \%$ & 51 \\
\hline \multirow{4}{*}{$T_{1}$} & 1 & $1,1765 \mu \mathrm{s}$ & $0,001317 \mu \mathrm{s}$ & 51 \\
\hline & 2 & $1,1779 \mu \mathrm{s}$ & $0,001326 \mu \mathrm{s}$ & 51 \\
\hline & 3 & $1,1754 \mu \mathrm{s}$ & $0,001169 \mu \mathrm{s}$ & 51 \\
\hline & 4 & $1,1733 \mu \mathrm{s}$ & $0,001564 \mu \mathrm{s}$ & 51 \\
\hline \multirow{4}{*}{$T_{2}$} & 1 & $53,694 \mu \mathrm{s}$ & $0,08925 \mu \mathrm{s}$ & 51 \\
\hline & 2 & $53,989 \mu \mathrm{s}$ & $0,08512 \mu \mathrm{s}$ & 51 \\
\hline & 3 & $53,836 \mu \mathrm{s}$ & $0,09009 \mu \mathrm{s}$ & 51 \\
\hline & 4 & $53,824 \mu \mathrm{s}$ & $0,08270 \mu \mathrm{s}$ & 51 \\
\hline
\end{tabular}

(because it is $m_{1}=m_{2}=m_{3}=m_{4}=m_{5}=m_{6}$ ).

TABLE II. EXPERIMENTAL CHECK OF STABILITY OF THE RECURRENT SURGE GENERATOR

\begin{tabular}{|c|c|c|c|c|c|c|c|c|}
\hline \multirow{2}{*}{$V_{0}$} & \multicolumn{2}{|c|}{ Channel 1} & \multicolumn{2}{|c|}{ Channel 2} & \multicolumn{2}{|c|}{ Channel 3} & \multicolumn{2}{|c|}{ Channel 4} \\
\hline & $\bar{F}_{1}$ & $u_{1}$ & $\bar{F}_{2}$ & $u_{2}$ & $\bar{F}_{3}$ & $u_{3}$ & $\bar{F}_{4}$ & $u_{4}$ \\
\hline $300 \mathrm{~V}$ & 0,99924 & 0,00025 & 0,99676 & 0,00024 & 1,0063 & 0,00027 & 0,99781 & 0,00025 \\
\hline $250 \mathrm{~V}$ & 0,99994 & 0,00016 & 0,99792 & 0,00011 & 1,0073 & 0,00019 & 0,99895 & 0,00018 \\
\hline $200 \mathrm{~V}$ & 1,0011 & 0,00033 & 0,99853 & 0,00036 & 1,0078 & 0,00028 & 0,99938 & 0,00033 \\
\hline $150 \mathrm{~V}$ & 0,99965 & 0,00018 & 0,99785 & 0,00014 & 1,0063 & 0,00015 & 0,99831 & 0,00016 \\
\hline $100 \mathrm{~V}$ & 1,0048 & 0,00026 & 1,0033 & 0,00028 & 1,0125 & 0,00025 & 1,0040 & 0,00019 \\
\hline $50 \mathrm{~V}$ & 1,0143 & 0,00033 & 1,0102 & 0,00031 & 1,0214 & 0,00032 & 1,0132 & 0,00032 \\
\hline$F$ & \multicolumn{2}{|c|}{1,0032} & \multicolumn{2}{|c|}{1,0008} & \multicolumn{2}{|c|}{1,0103} & \multicolumn{2}{|c|}{1,0019} \\
\hline $\boldsymbol{u}_{\mathrm{B} 0}$ & \multicolumn{2}{|c|}{0,0064} & \multicolumn{2}{|c|}{0,0054} & \multicolumn{2}{|c|}{0,0063} & \multicolumn{2}{|c|}{0,0065} \\
\hline$u_{\mathrm{F}}$ & \multicolumn{8}{|c|}{0,0062} \\
\hline
\end{tabular}
AND THE MEASURING CHANNELS

TABLE III. EXPERIMENTAL CHECK OF THE NONLINEARITY OF THE RECURRENT SURGE GENERATOR AND MEASURING CHANNELS 
The measured relative standard uncertainty due to the nonlinearity of all channels is negligibly small. However, when estimating the total (overall) measurement uncertainty of voltage ratio, standard uncertainty of calibration of HiRES transient recorder should be taken into account $(0.32 \%$ according to transient recorder calibration certificate).

It is reasonable to change the sensitivity of measuring channel (V/div) to achieve higher resolution when measuring lower response voltages. Therefore, it was necessary to examine how changing the range (sensitivity) of the measuring channel affects the repeatability of voltage ratio measurement. For this purpose, a relatively low voltage of approximately $60 \mathrm{~V}$ is measured by measuring channels with a sensitivity change: $10 \mathrm{~V} / \mathrm{div}, 20 \mathrm{~V} / \mathrm{div}$, and $50 \mathrm{~V} / \mathrm{div}$. The measurements were repeated 10 times and the results are presented in Table IV.

TABLE IV. INFLUENCE OF MEASURING CHANNEL SENSITIVITY ON MEASUREMENT REPEATABILITY

\begin{tabular}{|c|c|c|c|c|c|c|c|c|}
\hline \multirow{2}{*}{ Sensitivity } & \multicolumn{2}{|c|}{ Channel 1} & \multicolumn{2}{|c|}{ Channel 2} & \multicolumn{2}{|c|}{ Channel 3} & \multicolumn{2}{|c|}{ Channel 4} \\
\hline & $\bar{V}_{1} / \mathrm{V}$ & $s d_{1} / \%$ & $\bar{V}_{2} / \mathrm{V}$ & $s d_{2} / \%$ & $\bar{V}_{3} / \mathrm{V}$ & $s d_{3} / \%$ & $\bar{V}_{4} / \mathrm{V}$ & $s d_{4} / \%$ \\
\hline $10 \mathrm{~V} / \mathrm{div}$ & 60,292 & 0,020 & 59,965 & 0,020 & 60,159 & 0,020 & 59,929 & 0,028 \\
\hline $20 \mathrm{~V} / \mathrm{div}$ & 60,362 & 0,10 & 59,943 & 0,073 & 60,194 & 0,070 & 59,995 & 0,059 \\
\hline $50 \mathrm{~V} / \mathrm{div}$ & 60,592 & 0,12 & 60,035 & 0,12 & 60,421 & 0,132 & 60,178 & 0,10 \\
\hline$\overline{\bar{V}} / \mathrm{V}$ & \multicolumn{2}{|c|}{60,415} & \multicolumn{2}{|c|}{59,981} & \multicolumn{2}{|c|}{60,258} & \multicolumn{2}{|c|}{60,034} \\
\hline$s d / \%$ & \multicolumn{2}{|c|}{0,26} & \multicolumn{2}{|c|}{0,080} & \multicolumn{2}{|c|}{0,24} & \multicolumn{2}{|c|}{0,21} \\
\hline$r$ & \multicolumn{2}{|c|}{3} & \multicolumn{2}{|c|}{3} & \multicolumn{2}{|c|}{3} & \multicolumn{2}{|c|}{3} \\
\hline$s d_{\text {pool }} / \%$ & \multicolumn{8}{|c|}{0,21} \\
\hline
\end{tabular}

The relative standard uncertainty due to change in vertical scale sensitivity for all four channels, $s d_{\text {pool }} / \%$, is calculated as the pooled standard deviation (35).

\section{MEASUREMENT UNCERTAINTY WITH NEW PROCEDURE}

The analysis made in previous chapters confirmed the assumption that new procedure gives less measurement uncertainty of voltage ratio. Time intervals are measured with the same uncertainty as with the old procedure.

Since both voltages (applied voltage and response voltage) are measured by the same measuring channel, the total (overall) uncertainty of voltage ratio measured by the new procedure is reduced to the components of the stability of generator and measuring channel $u_{\mathrm{st}}$, nonlinearity of the measuring channel $u_{\mathrm{nl}}$, the change of sensitivity of measuring channel $u_{\text {se }}$ and component of software uncertainty $u_{\text {soft }}$ :

$$
\dot{u}_{\mathrm{T}}\left(v_{\% i}\right)=\sqrt{u_{\% s t}^{2}+u_{\% n l}^{2}+u_{\% s e}^{2}+2 \cdot u_{\% s o f t}^{2}}
$$

The first three components are characteristic of the measuring system in use. Based on the measurements made, the overall measurement uncertainty for our measuring system is:

$$
\dot{u}_{\mathrm{T}}\left(v_{\% i}\right)=\sqrt{0,11^{2}+0,32^{2}+0,21^{2}+2 \cdot 0,058^{2}}=0,4066 \%
$$

With the new measurement procedure, the total (overall) uncertainty of response voltage expressed in percentage of the applied voltage is $0,4066 \%$, which is about half the uncertainty when using the old procedure.

Complete measurement result of voltage ratio when using the new procedure for example 1 is:

$$
v_{\% i}=v_{\% i} \pm 2 \cdot \dot{u}_{\mathrm{T}}\left(v_{\% i}\right)=\frac{46,66}{300,3} 100 \% \pm 2 \cdot 0,4066 \%=\{15,54 \pm 0,81\} \%
$$

Complete measurement result of time interval is estimated in the same way as in the old procedure (see Examle 2).

The measurement uncertainty estimates of the new procedure are verified by extensive repetition of the measurements $(r=51)$ extended to two days. The results are shown in Table V.

It can be seen that the standard uncertainty of repeatability for all repeated measurements at all levels of the selected voltage ratios is much smaller than the estimated overall measurement uncertainty according to (36). 
TABLE V. EXPERIMENTAL CHECK OF NEW PROCEDURE SUITABILITY BY REPEATING THE MEASUREMENT $(r=51)$ AT DIFFERENT VOLTAGE LEVELS

\begin{tabular}{|c|c|c|c|c|c|c|c|c|}
\hline & \multicolumn{2}{|c|}{ Channel 1} & \multicolumn{2}{|c|}{ Channel 2} & \multicolumn{2}{|c|}{ Channel 3} & \multicolumn{2}{|c|}{ Channel 4} \\
\hline & $\bar{X}_{1}$ & $u_{1} / \%$ & $\bar{X}_{2}$ & $u_{2} / \%$ & $\bar{X}_{3}$ & $u_{3} / \%$ & $\bar{X}_{4}$ & $u_{4} / \%$ \\
\hline$V_{0} / \mathbf{V}$ & 302,52 & 0,06725 & 300,76 & 0,1067 & 301,73 & 0,05860 & 301,27 & 0,1104 \\
\hline$V_{1} / \mathrm{V}$ & 276,13 & 0,08413 & 274,60 & 0,1061 & 275,33 & 0,07241 & 274,88 & 0,1080 \\
\hline$\left(\frac{V_{1}}{V_{0}}\right) / \%$ & 91,277 & 0,09773 & 91,303 & 0,09435 & 91,251 & 0,08033 & 91,241 & 0,08862 \\
\hline$\beta / \%$ & $-0,24012$ & 0,04652 & $-0,24778$ & 0,04967 & $-0,21083$ & 0,04427 & $-0,18255$ & 0,04516 \\
\hline$T_{1} / \mu \mathrm{S}$ & 1,1765 & 0,001317 & 1,1779 & 0,001326 & 1,1754 & 0,001169 & 1,1733 & 0,001564 \\
\hline \multirow[t]{3}{*}{$T_{2} / \mu s$} & 53,694 & 0,08925 & 53,989 & 0,08512 & 53,836 & 0,09009 & 53,824 & 0,08270 \\
\hline & \multicolumn{2}{|c|}{ Channel 1} & \multicolumn{2}{|c|}{ Channel 2} & \multicolumn{2}{|c|}{ Channel 3} & \multicolumn{2}{|c|}{ Channel 4} \\
\hline & $\bar{X}_{1}$ & $u_{1} / \%$ & $\bar{X}_{2}$ & $u_{2} / \%$ & $\bar{X}_{3}$ & $u_{3} / \%$ & $\bar{X}_{4}$ & $u_{4} / \%$ \\
\hline$V_{0} / \mathbf{V}$ & 302,34 & 0,09113 & 300,77 & 0,08319 & 301,66 & 0,07919 & 301,17 & 0,08576 \\
\hline$V_{1} / \mathrm{V}$ & 199,10 & 0,1778 & 198,03 & 0,1623 & 198,66 & 0,1542 & 198,33 & 0,1654 \\
\hline$\left(\frac{V_{1}}{V_{0}}\right) / \%$ & 65,853 & 0,1157 & 65,841 & 0,1122 & 65,854 & 0,1161 & 65,854 & 0,1074 \\
\hline$\beta / \%$ & $-0,24818$ & 0,06734 & $-0,24296$ & 0,06141 & $-0,22620$ & 0,06096 & $-0,19285$ & 0,06422 \\
\hline$T_{1} / \mu s$ & 1,1733 & 0,002324 & 1,1751 & 0,002402 & 1,1723 & 0,002123 & 1,1704 & 0,002314 \\
\hline \multirow[t]{3}{*}{$T_{2} / \mu s$} & 53,817 & 0,1187 & 54,059 & 0,1034 & 53,889 & 0,1130 & 53,913 & 0,1132 \\
\hline & \multicolumn{2}{|c|}{ Channel 1} & \multicolumn{2}{|c|}{ Channel 2} & \multicolumn{2}{|c|}{ Channel 3} & \multicolumn{2}{|c|}{ Channel 4} \\
\hline & $\bar{X}_{1}$ & $u_{1} / \%$ & $\bar{X}_{2}$ & $u_{2} / \%$ & $\bar{X}_{3}$ & $u_{3} / \%$ & $\bar{X}_{4}$ & $u_{4} / \%$ \\
\hline$V_{0} / \mathbf{V}$ & 302,59 & 0,03461 & 300,69 & 0,03989 & 301,81 & 0,03655 & 301,11 & 0,03042 \\
\hline$V_{1} / \mathbf{V}$ & 98,009 & 0,08405 & 97,378 & 0,07784 & 97,741 & 0,07949 & 97,519 & 0,08307 \\
\hline$\left(\frac{V_{1}}{V_{0}}\right) / \%$ & 32,391 & 0,03125 & 32,384 & 0,02796 & 32,385 & 0,02576 & 32,386 & 0,02780 \\
\hline$\beta / \%$ & $-0,23447$ & 0,02568 & $-0,24051$ & 0,02874 & $-0,19131$ & 0,03094 & $-0,17504$ & 0,02652 \\
\hline$T_{1} / \mu s$ & 1,1750 & 0,001056 & 1,1767 & 0,001134 & 1,1749 & 0,001027 & 1,1720 & 0,001194 \\
\hline \multirow[t]{3}{*}{$T_{2} / \mu \mathrm{s}$} & 53,746 & 0,02312 & 53,989 & 0,03036 & 53,807 & 0,02353 & 53,847 & 0,02209 \\
\hline & \multicolumn{2}{|c|}{ Channel 1} & \multicolumn{2}{|c|}{ Channel 2} & \multicolumn{2}{|c|}{ Channel 3} & \multicolumn{2}{|c|}{ Channel 4} \\
\hline & $\bar{X}_{1}$ & $u_{1} / \%$ & $\bar{X}_{2}$ & $u_{2} / \%$ & $\bar{X}_{3}$ & $u_{3} / \%$ & $\bar{X}_{4}$ & $u_{4} / \%$ \\
\hline$V_{0} / \mathrm{V}$ & 302,32 & 0,05412 & 300,32 & 0,06692 & 301,59 & 0,05606 & 300,79 & 0,06783 \\
\hline$V_{1} / \mathbf{V}$ & 46,633 & 0,05415 & 46,392 & 0,07746 & 46,515 & 0,05742 & 46,356 & 0,06875 \\
\hline$\left(\frac{V_{1}}{V_{0}}\right) / \%$ & 15,425 & 0,01162 & 15,447 & 0,01233 & 15,423 & 0,01048 & 15,411 & 0,01109 \\
\hline$\beta / \%$ & $-0,23862$ & 0,03844 & $-0,24757$ & 0,03530 & $-0,20571$ & 0,03232 & $-0,18921$ & 0,03624 \\
\hline$T_{1} / \mu s$ & 1,1769 & 0,001342 & 1,1783 & 0,001309 & 1,1755 & 0,001118 & 1,1733 & 0,001286 \\
\hline$T_{2} / \mu \mathrm{S}$ & 53,672 & 0,04594 & 53,934 & 0,04574 & 53,800 & 0,05341 & 53,790 & 0,04503 \\
\hline
\end{tabular}

\section{CONCLUSION}

Measurement uncertainty is a measure of the quality of a measurement result. Knowledge of measurement uncertainty is necessary for the acceptance of a measurement result and it is a basic parameter in the selection of measurement equipment and methods, as well as the basis for correct and reliable decisions.

By using measurement uncertainty analysis of LI voltage ratio, a better measurement procedure is developed.

The experimental check of the proposed new procedure shows that the amplitude (voltage) uncertainty has been reduced to half, with reducing the measurement time for about $15 \%$ when measured with 4-channel oscilloscope, or about $40 \%$ when measured with 2-channel oscilloscope. 


\section{REFERENCES}

[1] IEC 60060-2:2010, High-voltage techniques Part 2: Measuring systems

[2] IEC 61083-2:2013, Instruments and software used for measurement in high-voltage and high-current tests, Part 2: Requirements for software for tests with impulse voltages and currents

[3] Guide to the expression of uncertainty in measurement (GUM), ISO, 1993., ISBN 92-67-10188-9, International Organization of standardization, 1993

[4] 10X Passive Probe P6139B, Tektronix, Instructions

[5] Tektronix, Service Manual, TDS7000 Series Digital Phosphor Oscilloscopes (TDS7104 \& TDS7054), 071-0711-03

[6] Recurrent Surge Generator Type 481, Haefely E 119.2 